\title{
Anderson localization in atomic mixtures
}

\author{
K. Ziegler ${ }^{1}$, O. Fialko ${ }^{2}$ \\ ${ }^{1}$ Institut für Physik, Universität Augsburg, D-86135 Augsburg, Germany \\ ${ }^{2}$ Centre for Theoretical Chemistry and Physics, Massey University (Albany Campus), Private \\ Bag 102904, North Shore MSC, Auckland 0745, New Zealand \\ E-mail: klaus.ziegler@physik.uni-augsburg.de
}

\begin{abstract}
A mixture of two types of atoms in an optical lattice is studied. Assuming that one type of atoms is much heavier than the other, the complex interplay of the two species leads to a correlated distribution of heavy atoms and to diffusion or Anderson localization of the light atoms. The latter depends on the distribution of the heavy atoms, where diffusion appears only at low temperatures.
\end{abstract}

\section{Introduction}

In search for a physical system where we can manipulate disorder systematically, a gas of ultracold atoms turns out to be a good candidate. Ultracold atoms in an optical or magnetic trap have been used to simulate various many-body systems. The main advantage of the trapped ultracold gas is that most of the physical parameters can be tuned easily during the experiment. First of all, the quantum statistics, i.e. bosons or fermions, can be chosen by using the proper atomic species. For instance, ${ }^{87} \mathrm{Rb}$ atoms are bosons and ${ }^{6} \mathrm{Li},{ }^{40} \mathrm{~K}$ atoms are fermions. The interaction between the atoms can be changed from attraction to repulsion by an external magnetic field due to a Feshbach resonance $[1,2]$. The underlying lattice structure of a solidstate system can be provided in the atomic system by an optical lattice [4]. In such a structure the atoms tunnel from one potential well to a neighboring one just like the electrons in a metal. On the other hand, the optical potential, which is created by counter-propagating laser fields, can easily be modified in strength by changing the laser intensity. Consequently, the tunneling rate of the atoms can be tuned by the latter. This has been used to study the Bose-Einstein condensate to Mott state transition in a Bose gas, where a Bose-Einstein condensate appears at large tunneling rates and a Mott phase with commensurate filling of the optical lattice at small tunneling rates [4].

Disorder in statistical or solid-state physics usually means that particles are scattered by a disordered (i.e. a randomly produced) array of other particles. This disordered array is static and does not change during the course of an experiment. For instance, the scattering of electrons in a crystal with randomly distributed impurity atoms is a typical realization of disordered physics. A comparable situation can be constructed in atomic systems subject to an optical lattice. The periodic structure of the optical lattice is normally quite robust such that disorder in the sense of impurities cannot easily be produced. However, in contrast to a crystal in solid-state physics, two or more different types of atoms can be mixed and brought in an optical lattice, where one type of the atoms is relatively light (e.g. ${ }^{6} \mathrm{Li}$ ) and the other type(s) is (are) heavy (e.g. ${ }^{40} \mathrm{~K}$, ${ }^{23} \mathrm{Na}[5],{ }^{87} \mathrm{Rb}$ ). The different masses lead to different dynamical properties. In particular, the 
heavy atoms behave almost like static particles, whereas the light atoms tunnel freely in the optical lattice. Consequently, the heavy atoms play the role of the impurity atoms in the crystal and the light atoms the role of the electrons. Thus disorder physics can be studied in mixtures of atoms which are available in experiments [6].

Disordered systems have attracted a lot of attention because they provide a new class of physics, including new phases and new types of phase transitions. The main reason is that scattering of quantum particles by a periodic structure is qualitatively different from scattering by a disordered structure: while the former is governed by the Bloch theorem, the latter experiences diffusion (for weak disorder) or even Anderson localization for strong disorder [7]. There is a phase transition between the diffusion-controlled regime and the localized regime $[8,9]$.

A fundamental concept for a theoretical description of disordered system is that physical quantities are averaged with respect to a statistical distribution of the random structures. It will be discussed in this article that averaging over thermal fluctuations of heavy atoms provides such a disorder (or quenched) average of physical quantities, like the density or the mean-square displacement of light atoms.

\section{Model}

The prototype of fermionic many-body lattice systems is the Hubbard model which describes tunneling of spin-1/2 fermions (e.g. electrons) between nearest-neighbor lattice sites and a local interaction. This model can be generalized to describe the interaction of spinless fermion atoms with localized atoms as

$$
H=-t_{f} \sum_{\left\langle r, r^{\prime}\right\rangle} f_{r}^{\dagger} f_{r^{\prime}}+\sum_{r}\left[-\mu_{b} b_{r}^{\dagger} b_{r}-\mu_{f} f_{r}^{\dagger} f_{r}+U_{b f} b_{r}^{\dagger} b_{r} f_{r}^{\dagger} f_{r}+U_{b b} b_{r}^{\dagger} b_{r}^{\dagger} b_{r} b_{r}\right] .
$$

Here $f^{\dagger}(f)$ are creation (annihilation) operators of the light fermionic atoms, $b^{\dagger}(b)$ are the corresponding operators of the heavy atoms (fermions or bosons). The interaction between the light fermions at low temperatures is controlled by the Pauli principle, whereas the interaction between light fermions and the heavy atoms is controlled by $U_{b f}$ and the interaction between the heavy atoms by $U_{b b}$. The chemical potentials $\mu_{b}$ and $\mu_{f}$ controls the number of heavy and light atoms, respectively.

A grand-canonical ensemble is defined by the partition function, which is the trace of Boltzmann weights over all realizations of heavy and light atoms in the system:

$$
Z=\operatorname{Tr}_{b} \operatorname{Tr}_{f} e^{-\beta H} \text {. }
$$

Since there is no tunneling for the heavy atoms, they are characterized only by integer occupation numbers: for bosons $n_{r}=0,1,2,3, \ldots$ and for fermions due to the Pauli principle $n_{r}=0,1$. After performing the trace over the light fermions we arrive at the partition function

$$
Z=\sum_{\left\{n_{r}\right\}} e^{\beta \sum_{r}\left[\mu_{b} n_{r}-U_{b b} n_{r}\left(n_{r}-1\right)\right]} \operatorname{det}\left[\mathbf{1}+e^{-\beta h_{f}}\right],
$$

where $h_{f}$ is a matrix with respect to the lattice sites that describes tunneling of light atoms and includes a one-particle potential. The matrix elements are $\left(h_{f}\right)_{r, r^{\prime}}=-\hat{t}_{r, r^{\prime}}+\left(U_{b f} n_{r}-\mu_{f}\right) \delta_{r, r^{\prime}}$.

\subsection{Characterization of heavy atoms}

According to Eq. (3) the heavy particles are distributed according to the probability distribution

$$
P\left(\left\{n_{r}\right\}\right)=\frac{1}{Z} e^{\beta \sum_{r}\left[\mu_{b} n_{r}-U_{b b} n_{r}\left(n_{r}-1\right)\right]} \operatorname{det}\left[\mathbf{1}+e^{-\beta h_{f}}\right],
$$

with $\sum_{\left\{n_{r}\right\}} P\left(\left\{n_{r}\right\}\right)=1$. Thus the density of heavy atoms at lattice site $r$ is given by $\left\langle n_{r}\right\rangle_{b}=\sum_{\left\{n_{r}\right\}} n_{r} P\left(\left\{n_{r}\right\}\right)$. 


\subsection{Characterization of light atoms}

The characterization of the light atoms is controlled by quantum effects (i.e. tunneling between the potential wells of the optical lattice) and the interaction with the classical heavy atoms. Thus we need to describe the dynamics of the light atoms by the evolution of their quantum state.

In general terms, we consider a quantum gas in the initial equilibrium state $\left|\Psi_{0}\right\rangle$ and create a new state by adding a light atom at site $0: c_{0}^{\dagger}\left|\Psi_{0}\right\rangle$. Then we follow the evolution of the state with the additional particle, controlled by the Hamiltonian $H$, until the time $t$, where we get the (non-equilibrium) state $\left|\Phi_{t}\right\rangle=e^{-i H t} c_{0}^{\dagger}\left|\Psi_{0}\right\rangle$. From this we obtain for the mean-square displacement

$$
\sum_{r} r^{2}\left\langle\Phi_{t}\left|c_{r}^{\dagger} c_{r}\right| \Phi_{t}\right\rangle=\sum_{r} r^{2}\left\langle\Psi_{0}\left|c_{0} e^{i H t} c_{r}^{\dagger} c_{r} e^{-i H t} c_{0}^{\dagger}\right| \Psi_{0}\right\rangle
$$

Instead of using a fixed state $\left|\Psi_{0}\right\rangle$ we average with respect to a Boltzmann ensemble at inverse temperature $\beta$ of energy eigenstates:

$$
\left\langle r^{2}\right\rangle=\sum_{r} r^{2} \frac{\sum_{k} e^{-\beta E_{k}}\left\langle E_{k}\left|c_{0} e^{i H t} c_{r}^{\dagger} c_{r} e^{-i H t} c_{0}^{\dagger}\right| E_{k}\right\rangle}{\sum_{k} e^{-\beta E_{k}}}=\frac{1}{Z} \sum_{r} r^{2} \operatorname{Tr}\left[e^{-\beta H} c_{0} e^{i H t} c_{r}^{\dagger} c_{r} e^{-i H t} c_{0}^{\dagger}\right] .
$$

where the right-hand side can also be expressed by the density of light atoms $N_{r}$ as $\sum_{r} r^{2} N_{r}$. For long times and weak coupling $U_{b f}$ (or low temperatures) diffusion is expected with $\left\langle r^{2}\right\rangle \sim D t$ and the diffusion coefficient $D$. On the other hand, for stronger coupling (or higher temperatures) Anderson localization is expected. Thus there is no expansion at all, and the mean-square displacement is finite for $t \sim \infty$. Intermediate regimes with sub-diffusive behavior are also possible, where $\left\langle r^{2}\right\rangle \sim D t^{\alpha}$ with $0<\alpha<1$.

Returning to our specific case of the atomic mixture, we can separate the heavy atoms and the light atoms as in the partition function (3). This allows us to write for the mean-square displacement [10]

$$
\begin{aligned}
& \left\langle r^{2}\right\rangle=\frac{1}{Z} \sum_{\left\{n_{r}\right\}} e^{\beta \sum_{r}\left[\mu_{b} n_{r}-U_{b b} n_{r}\left(n_{r}-1\right)\right]} \operatorname{det}\left[\mathbf{1}+e^{-\beta h_{f}}\right] \\
& \times \sum_{r} r^{2}\left[e^{i t h_{f}}\left(\mathbf{1}+e^{-\beta h_{f}}\right)^{-1}\right]_{0 r}\left[e^{-i t h_{f}}\left(\mathbf{1}+e^{-\beta h_{f}}\right)^{-1}\right]_{r 0} .
\end{aligned}
$$

In the density $\left\langle n_{r}\right\rangle_{b}$ and in the mean-square displacement there is the distribution density of Eq. (4) as a common non-negative factor. This implies that density of light atoms $N_{r}$ and their mean-square displacement $\left\langle r^{2}\right\rangle=\sum_{r} r^{2} N_{r}$ appear as expectation values with respect to the distribution density of Eq. (4) as

$$
N_{r}=\left\langle\left[e^{i t h_{f}}\left(\mathbf{1}+e^{-\beta h_{f}}\right)^{-1}\right]_{0 r}\left[e^{-i t h_{f}}\left(\mathbf{1}+e^{-\beta h_{f}}\right)^{-1}\right]_{r 0}\right\rangle_{b} .
$$

Thus the distribution of heavy atoms is a realization of quenched correlated disorder.

Using the single-particle Green's function

$$
\mathcal{G}_{r r^{\prime}}(t)=\left[e^{-i t h_{f}}\left(\mathbf{1}+e^{-\beta h_{f}}\right)^{-1}\right]_{r r^{\prime}},
$$

we obtain $N_{r}=\left\langle\mathcal{G}_{0 r}^{\dagger}(t) \mathcal{G}_{r 0}(t)\right\rangle_{b}$. For a given configuration $\left\{n_{r}\right\}$ of heavy atoms the Green's function can also be expressed by eigenfunctions of the single-particle Hamiltonian $h_{f}\left(h_{f} \phi_{k}=\right.$ $e_{k} \phi_{k}$ ). The spatial properties of these eigenfunctions determine the spreading of the average particle density $N_{r}$ through the Green's function:

$$
\mathcal{G}_{r 0}(t)=\sum_{k} e^{-i e_{k} t} \frac{\phi_{k, r}^{*} \phi_{k, 0}}{1+e^{-\beta e_{k}}} .
$$


The denominator represents the Fermi-Dirac function, reflecting the fact that our atoms are fermions. At low temperatures all states with $e_{k}>0$ (i.e. states with energy below the chemical potential according to the definition of $h_{f}$ ) contribute equally to the Green's function. According to the theory of Anderson localization, it can be assumed that $\left|\phi_{k, r}\right| \sim e^{-|r| / \xi_{k}}$, where $\xi_{k}$ is the localization length. After a Fourier transformation of the time-dependent density in Eq. (8), the $\omega=0$ Fourier component of $N_{r}$ reads

$$
\int_{0}^{\infty} N_{r} d t=\sum_{k} \frac{\left|\phi_{k, r}^{*} \phi_{k, 0}\right|^{2}}{\left(1+e^{-\beta e_{k}}\right)^{2}} \sim \frac{e^{-2|r| / \xi}}{\left(1+e^{-\beta e_{k_{0}}}\right)^{2}} \quad(r \sim \infty),
$$

where $\xi$ is the largest localization length and $e_{k_{0}}$ the corresponding energy level. Thus the expansion of the wave packet on large scales is controlled by $\xi$. This result suggests that the spatial expansion of an atomic cloud is governed by the largest length scale of the system.

\section{Strong-coupling expansion: classical Ising model}

In order to evaluate the distribution of heavy atoms and the localization length of the light atoms, we employ an approximation to the distribution function. The simplest case is a weakcoupling expansion for $U_{b f} / t_{f} \ll 1$. However, this leads only to a renormalized chemical potential [11] and is not very useful here. A strong-coupling expansion with $t_{f} / U_{b f} \ll 1$, on the other hand, provides an effective coupling of heavy atoms between nearest-neighbor potential wells $[11,12,13,14]$. In leading order of this expansion the determinant in Eq. (3) reduces to (cf. $[15])$

$$
\operatorname{det}\left[\mathbf{1}+e^{-\beta h_{f}}\right] \approx e^{\frac{\beta}{2} \sum_{\left\langle r, r^{\prime}\right\rangle} E\left(n_{r}, n_{r^{\prime}}\right)} \prod_{r}\left[1+e^{\beta A_{r}}\right]
$$

with $A_{r}=\mu_{f}-U_{b f} n_{r}$ and

$$
E\left(n_{r}, n_{r^{\prime}}\right)=\frac{e^{\beta A_{r}}-e^{\beta A_{r^{\prime}}}}{A_{r}-A_{r^{\prime}}} \frac{t_{f}^{2}}{\left(1+e^{\beta A_{r}}\right)\left(1+e^{\beta A_{r^{\prime}}}\right)} .
$$

Then the partition function in Eq. (3) reads

$$
Z \approx \sum_{\left\{n_{r}\right\}} e^{-\beta \sum_{r} F\left(n_{r}\right)} e^{\frac{\beta}{2} \sum_{\left\langle r, r^{\prime}\right\rangle} E\left(n_{r}, n_{r^{\prime}}\right)}
$$

where

$$
F(n)=-\frac{1}{\beta} \log \left[1+e^{\beta\left(\mu_{f}-U_{b f} n\right)}\right]-\left(\mu_{b}+U_{b b}\right) n+U_{b b} n^{2} .
$$

As the temperature is reduced, the bosons organize themselves in a low-energy state. Beginning with a small tunneling rate $t_{f}$ we can neglect the second factor in the Boltzmann weights of Eq. (14) and determine the number $\bar{n}$ which minimizes $F(n)$. At least one minimum exists because $F(n)$ increases monotonously for large values of $n$. Assuming that the value for the minimum of $F(n)$ is a number between the two integers $[\bar{n}]$ and $[\bar{n}]+1$, we study small fluctuations around $\bar{n}$ with $n_{r}=[\bar{n}]$ and $n_{r}=[\bar{n}]+1$. This enables us to rewrite the function $E\left(n_{r}, n_{r^{\prime}}\right)$, since only four different values are possible for $S_{r}=2\left(n_{r}-[\bar{n}]\right)-1$ with $S_{r}= \pm 1$ :

$$
E\left(n_{r}, n_{r^{\prime}}\right)=E_{0}+E_{1}\left(S_{r}+S_{r^{\prime}}\right)+E_{2} S_{r} S_{r^{\prime}},
$$

where the coefficients, using the short-hand notation $\epsilon\left(S, S^{\prime}\right)=E\left([\bar{n}]+(S+1) / 2,[\bar{n}]+\left(S^{\prime}+\right.\right.$ 1)/2)/4, are

$$
E_{0}=\epsilon(1,1)+\epsilon(-1,-1)+2 \epsilon(1,-1), \quad E_{1}=\epsilon(1,1)-\epsilon(-1,-1), \quad E_{2}=\epsilon(1,1)+\epsilon(-1,-1)-2 \epsilon(1,-1) .
$$



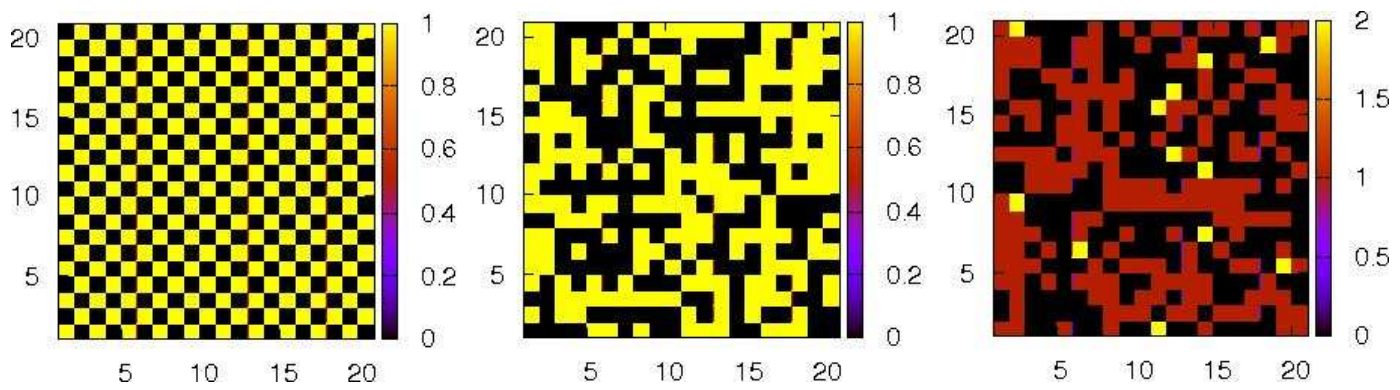

Figure 1. The distribution of heavy bosons at half-filling for $U_{b b}=U_{b f}=10 t_{f}$. The left plot is for $k_{B} T=0$, where bosons form perfect chess-board-like pattern. The middle plot is for $k_{B} T=2 t_{f}$, where the heavy bosons form chess-board-like clusters and clusters with more homogeneous patterns. The right plot is for $k_{B} T=8 t_{f}$ : at some lattice sites there are more than one boson as compared to the previous cases.

Eq. (16) provides an interpretation in terms of a classical Ising model for the spin $S_{r}=$ \pm 1 : $\quad E_{1}$ is proportional to an external magnetic field and $E_{2}$ is the nearest-neighbor spinspin coupling. Closer inspection reveals that the latter is negative, which indicates an antiferromagnetic coupling. This produces a staggered configuration with alternating $n_{r}=$ $[\bar{n}],[\bar{n}]+1$ occupation for small $E_{1}$. Then there is also competition between the antiferromagnetic spin-spin coupling and the magnetic field term $E_{1} S_{r}$, where the latter favors ferromagnetic order with homogeneous occupation. This implies a first-order transition between homogeneous and staggered (checkerboard in $d=2$ ) order of the heavy atoms. In the proximity of this first-order transitions there is phase separation, leading to the coexistence of homogeneous and staggered clusters. This is shown in the second plot of Fig. 1.

\section{Results and conclusions}

distribution of heavy atoms: The strong-coupling expansion, leading to an Ising model with antiferromagnetic coupling and an effective magnetic field, indicates already a complex distribution of heavy atoms. Now we employ a numerical approach to study the distribution in more detail and to evaluate the finite-size properties of localization length $\xi$ of the light fermions in Eq. (11). For this purpose we consider a two-dimensional lattice with $\mathcal{N}=N \times N(N=20)$ lattice sites and derive the statistically relevant realizations by Monte-Carlo sampling of the distribution of $\left\{n_{r}\right\}$. Examples at different temperatures are presented in Fig. 1.

expanding cloud of light atoms: An initial state of light atoms with high density at the center is prepared. Evolution under the effect of $e^{-i H t}$, as described in Sect. 2.2, leads either to diffusivelike expansion (cf. left panel in Fig. 2) or stays approximately in the initial state (cf. right panel in Fig. 2), depending on the strength of disorder created by the heavy atoms.

Localization transition of light atoms: The localization length $\xi$ is studied under the change of length scales of a finite lattice of length $L$ and width $M$ [9], representing an adiabatically expanding atomic cloud. In particular, we analyze the change of the localization length with respect to the width $M$. For this purpose, we define a reduced localization length of light atoms as $\Lambda_{M}=\xi / M$ and calculate this quantity by means of a numerical transfer-matrix approach for a given realization $\left\{n_{r}\right\}$ of heavy atoms [16]. For the latter statistically relevant realizations are chosen by Monte-Carlo sampling of the distribution of heavy atoms. Since for sufficiently large values of $L$ (we use typically $L \sim 10^{8}$ ) the localization length is presumably self-averaging, there is no need for additional averaging over different realizations. We use open boundaries along the action of the transfer matrix and periodic boundary conditions in the perpendicular direction. 

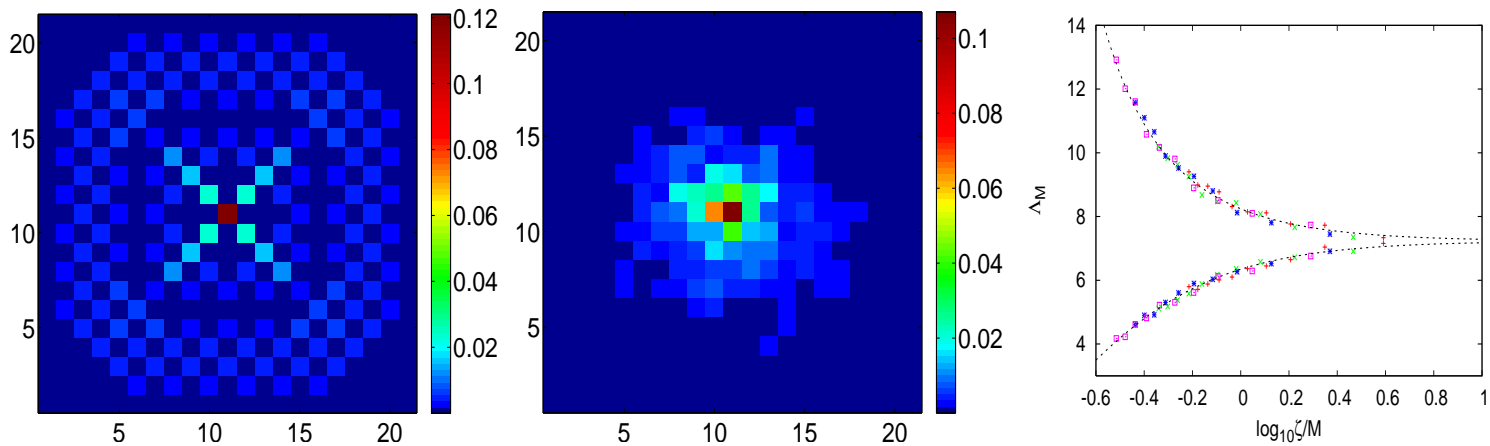

Figure 2. Evolution of a cloud of light atoms after $t=10 \hbar / t_{f}$. Left panel: expansion of the wave packet for weak disorder at $k_{B} T=0.05 t_{f}$. Middle panel: localization of the wave packet for strong disorder at $k_{B} T=0.2 t_{f}$. Right panel: Scaling of the localization length near the Anderson transition (from Ref. [18]).

$\Lambda_{M}$ either increases (delocalized states) or decreases (localized states) with the width $M$, depending on the system parameters (e.g. the inverse temperature $\beta$ ). There can also be a marginal behavior (e.g. for a special value $\beta_{c}^{\prime}$ ), where $\Lambda_{M}$ does not change with $M$. The latter indicates the existence of a phase transition from localized to delocalized states. A quantitative description of the behavior near $\beta_{c}^{\prime}$ can be based on the one parameter scaling hypothesis [9, 17]: Using the parameter $\zeta=\left|\beta-\beta_{c}^{\prime}\right|^{-\nu}$, we can fit our numerical data for $\Lambda_{M}$ quite well with

$$
\Lambda_{M}=\Lambda_{c} \exp \left[ \pm A\left(\frac{\zeta}{M}\right)^{-1 / \nu}\right]
$$

with $\Lambda_{c} \approx 10.9, A \approx 0.09$, and $\nu \approx 0.88$, as shown in the right panel of Fig. 2 [18].

From these results we can conclude that (i) a mixture of two atomic species creates a correlated distribution of heavy atoms and (ii) the light atoms undergo an Anderson transition, where localized (delocalized) states appear at high (low) temperatures.

[1] Fano U 1935 Nuovo Cimento 15612

[2] Feshbach H 1958 Ann. Phys. (N.Y.) 5357

[3] Kleppner D 2004 Physics Today 5712

[4] Greiner M, Mandel O, Esslinger T, Hänsch Th W and Bloch I 2002 Nature 41539

[5] Stan CA, Zwierlein MW, Schunck CH, Raupach SMF and Ketterle W 2004 Phys. Rev. Lett. 93143001

[6] DeMarco B and Jin DS 1999 Science 2851703

[7] Anderson PW 1958 Phys. Rev. 1091492

[8] Wegner F 1976 Z. Phys. B 25327

[9] Abrahams E, Anderson PW, Licciardello DC and Ramakrishnan TV 1979 Phys. Rev. Lett. 42673

[10] Ziegler K 2006, Laser Physics 16699

[11] Ates C and Ziegler K 2005 Phys. Rev. A 71063610

[12] Falicov LM and Kimball JC 1969 Phys. Rev. Lett. 22997

[13] Farkasovsky P 1997 Z. Phys. B 102 91; 2008 Europhysics Lett. 8437010

[14] Freericks JK and Zlatić V 2003 Rev. Mod. Phys. 751333

[15] Ziegler K and Schneider D 2005 J. Phys.: Condens. Matter 175489

[16] Pichard JL and Sarma G 1981 J. Phys. C 14 L127; Slevin K and Ohtsuki T 1999 Phys. Rev. Lett. 82382

[17] MacKinnon A and Kramer B 1983 Z. Phys. B 531

[18] Fialko O and Ziegler K 2009 Europhys. Lett. 85, 60003 